\title{
Grenzfall der Literatur?
}

\section{Freuds Kleiner Hans als linguistische Kriminalnovelle [1994]}

Als der in der naturwissenschaftlichen Medizin des 19. Jahrhunderts ausgebildete und ausgewiesene Arzt Sigmund Freud sich auf das Feld der für uns heute mit seinem Namen verbundenen Entdeckungen begab und zum Seelenforscher wurde, zum Psychoanalytiker, veröffentlichte er einen neuen Typ von Studien, seine Fallgeschichten, und dabei stellte sich heraus, dass der zünftige Neurophysiologe sich zum Schriftsteller gemausert hatte. Er selbst hat diesen Wandel mit einer gewissen Beunruhigung an sich beobachtet und 1895, in einer Studie zur Hysterie, ausgesprochen:

\footnotetext{
Ich bin nicht immer Psychotherapeut gewesen, sondern bin bei Lokaldiagnosen und Elektroprognostik erzogen worden wie andere Neuropathologen, und es berührt mich selbst noch eigentümlich, daß die Krankengeschichten, die ich schreibe, wie Novellen zu lesen sind, und daß sie sozusagen des ernsten Gepräges der Wissenschaftlichkeit entbehren. Ich muß mich damit trösten, daß für dieses Ergebnis die Natur des Gegenstandes offenbar eher verantwortlich zu machen ist als meine Vorliebe; Lokaldiagnostik und elektrische Reaktionen kommen bei dem Studium der Hysterie eben nicht zur Geltung, während eine eingehende Darstellung der seelischen Vorgänge, wie man sie vom Dichter zu erhalten gewohnt ist, mir gestattet, bei Anwendung einiger psychologischer Formeln doch eine Art von Einsicht in den Hergang einer Hysterie zu gewinnen. (Freud 1940ff., I: 227).
}

Auf der einen Seite also ,Lokaldiagnosen' und ,elektrische Reaktionen', kurz ,Neuropathologie‘, auf der anderen Seite ,Ich bin', ,es berührt mich', ,wie Novellen zu lesen', ,eingehende Darstellung der seelischen Vorgänge', ,eine Art von Einsicht'. Freud benennt hier nicht nur den Zwiespalt zwischen zwei grundverschiedenen Gattungen, sondern er führt auch jede in der ihr eigenen Sprache vor und stellt seinen Zwiespalt dar, indem er die beiden Stile, den kühlen objektiven, wissenschaftlichen, und den gefühlsverbundenen, persönlichen, dichterischen, ineinander verschränkt.

Seine Unruhe war begründet. Sie ist traditionsreich (vgl. zu diesem Thema Lepenies 1985). In der Geschichte der Wissenschaften lässt sich immer wieder beobachten, wie die Sprache an der Wirkung eines wissenschaftlichen Autors beteiligt ist und wie sie Wirkung verhindert. Mendels neue Vererbungslehre etwa wurde von seinen gelehrten Zuhörern in Brünn, denen er sie 1865 unter dem Titel „Experimente in der Pflanzenkreuzung“ vortrug, vielleicht schon deshalb verkannt, weil er sie durch einfache mathematische Statistiken verdeutlichte und sein Publikum, das im botanischen Zusammenhang auf Mathematik nicht gefasst war, abschweifte (vgl. Barber 1973). Die Wissenschaftsgeschichte scheint nicht 
arm an Beispielen dieser Art zu sein, es ließe sich geradezu als historische Regel formulieren, dass Sprache und Wirkung einander bedingen. Die Wahl der unerwarteten Gattung führt zur Apperzeptionsverweigerung: zum Sichverschließen, zur Unfähigkeit, das Dargebotene zu erkennen. Eine quer zur Erwartung liegende Ausdrucksweise kann die Aufnahme neuer wissenschaftlicher Erkenntnisse verhindern. Man ist anscheinend geneigt, zu verlangen, dass, auch was das Medium der Sprache, der Ausdrucksweisen und Gattungen angeht, alles in den gewohnten Geleisen weitergeht. Eingeführte Sprachen sind Bahnungen, Ordnungsformen, innerhalb deren einer sich möglichst zu halten hat. Ein Autor wähle die Gegenstände, die in sein Fach fallen, halte sich an die dafür vorgesehene Gattung und bediene sich der eingeführten Ausdrucksweisen! Unser Medium, die Sprache, wird hier als Zwischenwelt erkennbar, die an der Geschichte der Erkenntnisse einen selbständigen Anteil hat und als selbständiger Faktor der Wissenschaftsgeschichte Beachtung verdient. Eine engere Zusammenarbeit von Wissenschaftshistorikern und Wissenssoziologen mit Sprachhistorikern und Sprachsoziologen wäre sehr zu wünschen.

Wie verhält sich Freud im Widerstreit zwischen Wissenschaft und Dichtung? Nachdem er die Neurophysiologie hinter sich gelassen hat, lässt er sich auf Gegenstände ein, die wissenschaftlich bisher kaum ernst genommen worden sind, wie z.B. den Traum und das freie Assoziieren, den Witz und den Versprecher, und für die eine eingeführte wissenschaftliche Sprache nicht zur Verfügung steht. Das Unterbewusste fällt bis dahin am ehesten in die Observanz der Dichtung. Welche Sprache ist zu wählen?

An der zitierten Stelle schlägt er „eine eingehende Darstellung der seelischen Vorgänge, wie man sie vom Dichter zu erhalten gewohnt ist“, „bei Anwendung einiger psychologischer Formeln“ vor, also die Verbindung eines quasi naturwissenschaftlichen Sprachtyps mit dem literarischen. An diesem Rezept hat er festgehalten.

Im siebenten Kapitel der Traumdeutung beschreibt er die Psyche in der Art eines physikalischen Apparats (Freud 1940ff., II/III: 542ff.). Das Bauprinzip eines aus Linsensystemen zusammengesetzten Fernrohrs oder Mikroskops ist das allgemeine Modell, ist seine „Hilfsvorstellung“ für diese „Annäherung an etwas Unbekanntes“. Er verwendet Abkürzungssymbole, zeichnet Figuren, ganz wie der Autor eines physikalischen Lehrbuchs. Nicht nur der Begriff ,Apparat‘, auch seine Beschreibung hat den Charakter einer Analogie. - In anderen Kapiteln als gerade diesem siebenten ähnelt die Traumdeutung dagegen einer Konfession. Freud (1940ff., II/III: VIII) bereitet den Leser in der Vorbemerkung darauf vor:

Mit der Mitteilung meiner eigenen Träume aber erwies sich als untrennbar verbunden, daß ich von den Intimitäten meines psychischen Lebens fremden Einblicken mehr eröffnete, als 
mir lieb sein konnte und als sonst einem Autor, der nicht Poet, sondern Naturforscher ist, zur Aufgabe fällt. Das war peinlich, aber unvermeidlich.

Nicht Poet, sondern Naturforscher? In der Darstellung der Sexualtheorie treten die beiden Gattungen auseinander, die er in der Traumdeutung mischte. Auf der einen Seite, in den Drei Abhandlungen von 1905, wählt Freud die Sprache des versierten Biologen, er beginnt: „Die Tatsache geschlechtlicher Bedürfnisse bei Mensch und Tier drückt man in der Biologie durch die Annahme eines ,Geschlechtstriebes“ aus“ (Freud 1969ff., V: 47). Es folgt die Definition der Begriffe ,Sexualobjekt" und ,Sexualziel' und sodann ein erstes Kapitel Abweichungen in Bezug auf das Sexualobjekt, unterteilt in Unterkapitel, die wiederum in Unterabschnitte zerfallen usf. Eine strikte Systematik gliedert das Material. Das Verfahren ist deduktiv. Die allgemeinen Tatsachen werden vorangestellt und expliziert, die Termini eingeführt und dann mit ihnen gearbeitet. Der Autor verhält sich emotionslos neutral und lehnt jede ,Parteinahme‘ ab, ganz wie ein biologischer Systematiker, der eine noch unbekannte Art beschreibt. Die Tatsachen menschlichen ,Sexualverhaltens' werden vollständig beschrieben, auch wenn sie allgemein bekannt sind. Der Kuss, transformiert in den biologischen Sprachtyp, klingt z.B. so:

Es werden nämlich gewisse intermediäre (auf dem Weg zur Begattung liegende) Beziehungen zum Sexualobjekt, wie das Betasten und Beschauen desselben, als vorläufige Sexualziele anerkannt. Diese Betätigungen sind einerseits selbst mit Lust verbunden, andererseits steigern sie die Erregung, welche bis zur Erreichung des endgültigen Sexualzieles andauern soll. Eine bestimmte dieser Berührungen, die der beiderseitigen Lippenschleimhaut, hat ferner als Kuß bei vielen Völkern (die höchst zivilisierten darunter) einen hohen sexuellen Wert erhalten, obwohl die dabei in Betracht kommenden Körperteile nicht dem Geschlechtsapparat angehören, sondern den Eingang zum Verdauungskanal bilden. (Freud 1969ff., V: 60) ${ }^{1}$

Die Drei Abhandlungen zur Sexualtheorie sind als Ganzes, als Text, eine biologische Metapher. Auf der Gegenseite veröffentlicht Freud 1909, als Exempel für seine Sexualtheorie, die Analyse der Phobie eines fünfjährigen Knaben (Freud 1969ff., VIII: 9-123) und wählt dabei die Gattung einer linguistischen Kriminalnovelle. Dies zumindest ist die These. Sie hat drei Bestandteile:

1. Der Kleine Hans ist eine Kriminalgeschichte.

2. Freud führt einen linguistischen Indizienbeweis.

3. Die Darbietung dieser Fallgeschichte ähnelt einer Novelle.

1 Vgl. zum bisherigen den Aufsatz „Zur Terminologie der Psychoanalyse“ in diesem Band; Sulloway 1982. 


\section{1}

Freud liebt das Bild des ,Aufdeckens', er spricht auch am Beginn des Kleinen Hans vom „schichtweisen Aufdecken psychischer Bildungen“ (Freud 1969ff., VIII: 13). Man könnte an einen Archäologen denken oder auch an einen Detektiv. Der Vergleich des Analytikers mit einem Detektiv ist oft gezogen worden, zuletzt von Alfred Lorenzer, der in Edgar Alan Poe einen Ahnherrn des ,szenischen Verstehens' sieht. ${ }^{2}$

Mir drängt sich ein anderer Vergleich auf, wenn ich den ganzen Fallbericht vor Augen habe. Er ähnelt einer Gerichtsverhandlung, einem Prozess; die Parallele reicht weit.

Der ,Fall` ist bekanntlich dieser: Der kleine fast fünfjährige Hans hat plötzlich Angst, auf die Gasse zu gehen; er fürchtet, dass ihn ein Pferd beißen werde. Er ist weich gestimmt, weint, hat Sehnsucht nach der Mama und fürchtet, das Pferd werde ins Zimmer kommen. Das ist die nervöse Störung. Wir könnten auch sagen, das Krimen oder die Leiche oder der Tatbestand. Zugleich ist es das Rätsel, das, wie im Detektivroman, seiner Lösung harrt. Woher die Angst?

Die beteiligten Personen sind:

Der kleine Hans, im Alter von 3 bis 5 Jahren. Schon die mittelalterliche Poetik rät, einer Person eine einzige hervorstechende Eigenschaft zu geben. Hans ist vor allem ein kleiner Forscher; sein aufgewecktes Interesse gilt zunehmend dem Erotischen. Er hat ziemlich große Ähnlichkeit mit Freud.

Die schöne Mutter. Sie hat eine Schicksalsrolle, wie es heißt. Den Dreieinhalbjährigen bedroht sie einmal mit Kastration.

Der Vater. Er ist Arzt und ein Schüler Freuds, zeichnet Gespräche mit dem Jungen auf und stellt sie dem Professor zur Verfügung. In der Zeit der Krankheit hat er die Rolle des Untersuchungsrichters, verhört und befragt das Kind. Wie die Mutter setzt er dem Forscherdrang des Kleinen Schranken entgegen und gibt ihm unbefriedigende Antworten.

Die drei Genannten sind die Personen eines Familiendramas, das von Sophokles gedichtet worden ist.

Im Hintergrund hält sich Freud als der oberste Richter. Er nimmt die Protokolle des Kindesvaters entgegen, wägt die Indizien, hat die richterliche Tugend des Abwartens und der vorurteilslosen Aufmerksamkeit, einmal greift er in die Untersuchung ein und entschlüsselt den Fall. Diese höchste Instanz spricht auch

2 Lorenzer 1985. Zum Folgenden vgl. auch die aufschlussreiche Interpretation der Fallgeschichte des Kleinen Hans in Lorenzer 1970: 93-103. 
das Urteil. Sie ist allwissend und kann die Zukunft voraussehen. „Spricht denn der Professor mit dem lieben Gott, daß er das alles vorher wissen kann?" fragt der kleine Hans (Freud 1969ff., VIII: 41).

Die Rollenverteilung zwischen dem Vater des Kindes und Freud ist also die zwischen Vernehmungsrichter und oberstem Richter oder, wenn wir sie als Detektive sehen wollen, zwischen dem Assistenten, der voreilige Schlüsse zieht und gelegentlich falschen Spuren folgt, und dem Meisterdetektiv: Sherlock Holmes und Watson.

Das Opfer ist in diesem Stück zugleich der Täter. Auch das gehört zu den Voraussetzungen der sophokleischen Tragödie.

Die Aufklärung der Pferdephobie geschieht in drei Phasen:

1. Die Vorgeschichte des Angeklagten.

2. Der Prozess.

3. Das Urteil, mit der dazugehörigen Urteilsbegründung und abschließender Belehrung.

1. Die Vorgeschichte der Pferdeangst - bei Freud die Einleitung - ist eine der Zeit weit vorauseilende Studie zur begrifflichen Entwicklung eines Kindes zwischen dem 3. und 5. Lebensjahr, individuell und zugleich modellhaft. Hans ist voller Wissbegierde, er fragt und zieht Schlüsse, zweifelt und kommt voran, stufenweise erwirbt er sich wichtige Unterscheidungen. In dem Zeitabschnitt zwischen 21/2 und 43/4 Jahren begegnet der kleine Forscher einer Reihe ihm überwiegend unähnlicher Grundphänomene und setzt sich mit ihnen auseinander. Ihn beschäftigen besonders Kennzeichen und Vorgänge an leblosen Gegenständen oder am Tier, ihm tritt das andere Geschlecht gegenüber und die ,Großen“, die ihm etwas verbergen. Wenn man das in Freuds Darstellung Angelegte ein wenig systematisiert, kann man sagen, das Kind nähere sich einer Vorstellung von Klassen und Kategorien, wie sie in der analytischen Semantik als semantische Merkmale zur Charakterisierung von Wortinhalten verwendet werden, dies freilich in einem noch kindlich tastenden Stadium.

Der Held der Geschichte ist selbst ein kleiner Detektiv, insbesondere auf erotischem Gebiet. Er entpuppt sich im 4. Lebensjahr als vielseitiger ,Erotiker', hat Mäderln gern und einen Buben - Freud spricht von einer bemerkenswerten Beweglichkeit und polygamischen Veranlagung und von „Homosexualität“ -, er sucht die Mama zu verführen. „Unser kleiner Hans scheint wirklich ein Ausbund aller Schlechtigkeiten zu sein!“ kommentiert er (Freud 1969ff., VIII: 21).

Der Wissenstrieb des kleinen Hans stößt aber vorzeitig auf Schranken, die von den Erwachsenen, von der ihn umgebenden Kultur aufgerichtet werden. Die Erwachsenen von 1909 erkennen eine kindliche Sexualität nicht an bzw. sie untersagen sie z. T. drastisch und behindern die begriffliche Entwicklung des Kindes. 
Hans' Eltern enthalten ihm die Wahrheit über den Unterschied der Geschlechter vor und klären ihn, als das Schwesterchen geboren wird, über den Vorgang der Geburt nicht auf. Man erzählt ihm das Märchen vom Storch. Da er etwas ganz anderes zu beobachten meint, ruft das Unsicherheit bei ihm hervor, und macht es ihn misstrauisch. Am Ende der Vorgeschichte hat er etwas von seiner Unschuld verloren, er leugnet, ein Traum fällt nach Freud bereits in die erste Zeit der Verdrängung. Es spitzt sich etwas zu. Die Sprachregelung der Großen und seine eigene, unterdrückte Triebwelt sind auseinandergetreten, die Situation ist neuroseverdächtig. „Der Kernkomplex der Neurose findet sich auf diese Weise konstituiert“, heißt es in der parallelen Arbeit Über infantile Sexualtheorien (Freud 1969ff., V: 175).

Eindämmung eines Wissenstriebs also, Behinderung der begrifflichen Entwicklung als Beitrag zu einer Krankheitsdisposition - dies ist das Gedankenmodell, nach dem die Vorgeschichte aufgefasst und dargeboten ist. Damit ist die Exposition abgeschlossen, der Hauptteil des Dramas kann beginnen.

2. Der Prozess - bei Freud heißt der zweite Teil Krankengeschichte und Analyse - setzt abrupt ein (Freud 1969ff., VIII: 26).

„Geehrter Herr Professor! Ich sende Ihnen wieder ein Stückchen Hans, diesmal leider Beiträge zu einer Krankengeschichte.“ Von einer nervösen Störung ist die Rede, eben der Furcht, dass ihn auf der Gasse ein Pferd beißen werde, der Vater ist sehr beunruhigt und fragt nach dem Erreger der Störung. Es ist ein Rätsel. - Das Wort ,Rätsel` ist das Leitmotiv dieses Teils.

Nach diesem dramatischen Einsatz wird die unmittelbare Vorgeschichte nachgeholt, und danach wird Schicht um Schicht die psychische Bildung der Phobie aufgedeckt. Es gibt dabei Verirrungen und auch Durststrecken, die Fragen bzw. Verhöre des Vaters führen gelegentlich in die falsche Richtung, oder er ergeht sich in abwegigen Vermutungen. Die Hauptrichtung führt auf eine Lösung des Rätsels zu.

Es ist ein zweifacher Prozess, einer, der dem kleinen Hans gemacht wird, indem er zu immer neuen Geständnissen veranlasst wird, und einer, der in ihm in Gang gebracht wird und vorgeht und der immer neue Angstphantasien auf den Schirm wirft. In dem inneren Prozess wandert und verwandelt sich also die Angst. Sie gilt zuerst Pferden, eine Zeitlang Stellwagen, überhaupt schweren Wagen, und am Ende umfallenden Pferden, Pferden, die auf dem Rücken liegend „mit den Füßen Krawall machen“ (vgl. Freud 1969ff., VIII: 71, 79).

Der Prozess insgesamt bringt manche Hinweise auf den Täter, den Urheber der Phobie ans Tageslicht - von der linguistischen Indizienkette wird gleich genauer die Rede sein. Zu einer vollständigen, abgerundeten Aufklärung gelangt er noch nicht. Das Wichtigste ist indessen klar:

Die Angst erscheint als eine versetzte Angst. Sie gilt ursprünglich dem Vater und ist, in einer Art Abwehrmanöver, auf die Pferde verlagert. Diese sind ein Ersatzobjekt, das Bild des Vaters ist auf diese Weise frei von Angst. 
Die schweren Wagen symbolisieren nach Freud die Schwangerschaft der Mutter vor der Geburt des Schwesterchens.

Nach dem Durchgang durch diese und andere Ängste wird eine andere deutlich: die vor dem umfallenden Pferd. Hinter der Angst vor dem umfallenden Pferd verbirgt sich der Wunsch des Sohns, der Vater möchte umfallen oder sterben. Hans nämlich liebt seine Mutter. - Das Wort des Rätsels, das der die Protokolle kommentierende Erzähler und Richter Freud am Ende des Prozessgangs ausspricht, wird niemanden überraschen: „Der kleine Ödipus.“

Das Stück endet allerdings nicht tragisch, sondern opernhaft. Hans stellt sich vor, er sei mit der Mutter verheiratet und habe viele Kinder mit ihr, und den Vater, anstatt ihn zu beseitigen, ernennt er zum ,Großvatti“ und verheiratet auch ihn mit der eigenen Mutter. „Der kleine Ödipus hat eine glücklichere Lösung gefunden, als vom Schicksal vorgeschrieben ist“" (Freud 1969ff., VIII: 86).

Der Prozess ist eine Befreiung. Der kleine Forscher, der die Hemmung seines Forscherdranges mit einer Neurose bezahlen musste, ist am Ende wieder der aufgeweckte Detektiv, von dem sein wissenschaftlicher Erzeuger hofft, er werde „nur frühzeitig die Erfahrung machen, daß alles Wissen Stückwerk ist und daß auf jeder Stufe ein ungelöster Rest bleibt“ (Freud 1969ff., VIII: 88).

3. Der dritte Teil dieser Fallanalyse bringt die endgültige Aufklärung: das Urteil mit der dazugehörigen Urteilsbegründung und Belehrung. Freud nennt ihn „Epikrise“. ,Epikrise“ ist im medizinischen Sprachgebrauch die wissenschaftliche Beurteilung eines abgelaufenen Krankheitsfalles nach Entstehung, Verlauf und Ausgang.

Das Urteil lautet: Hans bestätigt „aufs grellste“, was Freud in den Drei Abhandlungen zur Sexualtheorie und ,auch schon in der Traumdeutung über die Sexualbeziehungen der Kinder zu den Eltern" ausgeführt hat (Freud 1969ff., VIII: 96). Er erfüllt vollständig den in diesem naturwissenschaftlichen Gesetzestext festgestellten Tatbestand. „Er ist wirklich ein kleiner Ödipus, der den Vater ,weg“, beseitigt haben möchte, um mit der schönen Mutter allein zu sein, bei ihr zu schlafen“ (Freud 1969ff., VIII: 96). Der gegen den Vater gerichtete Todeswunsch hat sich in Angst vor ihm verkehrt. Er ist in all diesem aber „kein Bösewicht“, sondern entspricht der allgemeinen menschlichen Konstitution. Das Urteil lautet daher: Freispruch. Hans wird von jeder Schuld entlastet (Freud 1969ff., VIII: 96f.).

Die folgende Urteilsbegründung stellt, wie etwa auch im Gerichtssaal, den Fall noch einmal dar, eine geklärte, auf die Grundlinien beschränkte und von den Irrwegen gereinigte Fassung des Prozesses. Dieser erscheint nun als folgerichtig. Die Details, die einzelnen Schritte der Analyse werden zusammengesetzt zu einem hochkomplexen und zugleich durchsichtigen Bild. Diese Synthese entspricht ganz dem Vorgehen des Richters in der Urteilsbegründung. 
Wenn jemandem Schuld zuzuweisen ist, dann den Eltern, die dem Kind Aufklärung vorenthalten haben. Das Buch schließt daher mit der gerichtstypischen Belehrung. Freud empfiehlt eine frühe Aufklärung, er meint, es hätten „die durch die Psychoanalyse gewonnenen Aufklärungen über die Herkunft der pathogenen Komplexe und über den Kern einer jeden Neurose eigentlich den Anspruch, vom Erzieher als unschätzbare Winke für sein Benehmen gegen das Kind gewürdigt zu werden“ (Freud 1969ff., VIII: 122). Die Kriminalgeschichte ist damit komplett.

\section{2}

Lässt sich auch nachweisen, dass wir es mit einer linguistischen Kriminalgeschichte zu tun haben? Gibt es eine linguistische Indizienkette in der Analyse des Kleinen Hans?

Alfred Lorenzer hat diese Frage in seinem bekannten Buch Sprachzerstörung und Rekonstruktion (1970) verneint. Die Absicht Lorenzers war es, Freuds naturwissenschaftliches Selbstverständnis, also das, was Habermas (1970: 263) als „,szientistisches Selbstmißverständnis der Psychoanalyse“ bezeichnet, durch ein Verständnis der Psychoanalyse als einer hermeneutischen Disziplin zu ersetzen. Er spricht von ,Tiefenhermeneutik ${ }^{*}$ und ersetzt die naturwissenschaftliche Bildersprache Freuds durch eine linguistische und hermeneutische Rede. Neurosen sind eine Form der ,Sprachzerstörung،, und im psychoanalytischen Prozess geschieht eine ,Rekonstruktion', Rekonstruktion im doppelten Sinne: als erinnerndes, stückweises und schichtweises Rekonstruieren des Vorgangs dieser Zerstörung, als Rekonstruktion des Tathergangs also, und als Wiederherstellung der unzerstörten anfänglichen Sprache. Der hermeneutische Schlüssel ist die Szene, das Gegenüber von Analytiker und Analysand. Es ermöglicht, durch das dramatische Verhältnis von Übertragung und Gegenübertragung, schichtweise zu Szenen in der Vergangenheit vorzudringen, die denen im Arztzimmer ähnlich sehen, und sie durch Analogie zu verstehen, bis hin zu der Urszene, mit der die Sprachzerstörung eingesetzt hat. Lorenzer unternimmt also den interessanten Versuch, das wissenschaftliche Verfahren der Psychoanalyse aus seiner naturwissenschaftlichen Verpuppung zu befreien und es in einer hermeneutischen Sprache darzustellen.

Als Beispiel wählt er Freuds Analyse des kleinen Hans, und er behauptet nun, dass in ihr ein Beispiel jenes szenischen Verstehens erkennbar werde, was ich nicht bestreite, dass aber in der Sprache des kleinen Hans die von ihm sogenannte Sprachzerstörung sich nicht bemerkbar mache (Lorenzer 1970: 97). Das bestreite ich. Freuds Analyse des kleinen Hans steckt m. E. voller sprachlicher Beobachtungen, und er zieht wichtige Schlüsse auf Grund von sprachlichen Befunden. 
Was Lorenzer Sprachzerstörung und ihre therapeutische Rekonstruktion nennt, hat zahlreiche linguistische Anhaltspunkte. Der Vater hat auffällige Formulierungen seines Kindes festgehalten.

Das beginnt mit der Vorgeschichte. Freuds Denkmodell ist, wie ausgeführt, eine Behinderung der begrifflichen Entwicklung des Kindes als Disposition für seine Erkrankung. Der kindliche Forscher erwirbt zwischen dem 3. und 5. Lebensjahr erste „Begriffe“, Vorstellungen von dem ihm Unähnlichen, Unterscheidungen zwischen dem Leblosen und Lebendigen, im Bezirk des Lebendigen zwischen Tieren und Menschen, im Bezirk der Menschen zwischen Großen und Kleinen, zwischen männlichen und weiblichen Wesen, d.h. die letztere Unterscheidung wird ihm zunächst vorenthalten, eben weil er noch klein ist.

Hans, kaum 3 Jahre: „Mama hast du auch einen Wiwimacher?“ Mama: „Selbstverständlich. Weshalb?“ Hans: „Ich hab nur gedacht.“ [...] Wenig später sieht er, wie eine Kuh gemolken wird: „Schau, aus dem Wiwimacher kommt Milch.“(Freud 1969ff., VIII: 14)

Der Ausgangspunkt der Beobachtung ist die eigene Person. „Das Ich bleibt der Maßstab, mit dem man die Welt ausmißt; durch beständiges Vergleichen mit der eigenen Person lernt man sie verstehen“ (Freud 1969ff., VIII: 93). Freud nimmt für diese Phase den ,egozentrischen' Standpunkt des Kindes an, jenen ,Egozentrismus', den dann Piaget (1972: 81) als ,eine Art systematischer und unbewußter Illusion, eine Art Perspektive“ für dieses Kindesalter beschrieben hat.

„Er sieht auf dem Bahnhofe, 33/4 Jahre alt, wie aus einer Lokomotive Wasser ausgelassen wird. ,Schau, die Lokomotive macht Wiwi. Wo hat sie denn den Wiwimacher?' Nach einer Weile setzt er nachdenklich hinzu: ,Ein Hund und ein Pferd hat einen Wiwimacher, ein Tisch und ein Sessel nicht.' So hat er ein wesentliches Kennzeichen für die Unterscheidung des Lebenden vom Leblosen gewonnen“, kommentiert Freud (1969ff., VIII: 16).

Hans setzt diese Kennzeichen nun bei allen Lebewesen voraus, beobachtet es am Pferd, erwartet es bei der Mama in pferdehafter Größe und ist, zumal sie seiner Erwartung nicht widerspricht, unfähig, diese Voraussetzung aufzugeben, als er sieht, wie seine kleine Schwester gebadet wird. Entgegen seiner Wahrnehmung sagt er: „Aber ihr Wiwimacher ist noch klein.“ „Wenn sie wächst, wird er schon größer werden“ (Freud 1969ff., VIII: 17f.).

Am Ende der Vorgeschichte steht diese Szene: „Hans (41/2 Jahre) sieht wieder $\mathrm{zu}$, wie seine kleine Schwester gebadet wird, und fängt an zu lachen. Man fragt ihn: ,Warum lachst du?' Hans: ,Ich lache über den Wiwimacher der kleinen Hanna. ' - ,Warum?` - ,Weil der Wiwimacher so schön ist.' Die Antwort ist natürlich eine falsche“, setzt Freud (1969ff., VIII: 25) hinzu.

Hans erkennt hier, Freud zufolge, erstmals den Unterschied der Geschlechter an, freilich nur durch sein Lachen. Das Lachen bestätigt den Erwerb einer Einsicht; 
auf der Ebene des Wortes leugnet Hans, was er lachend gestanden hat. Die außerverbale Mitteilung gilt, wenn sie im Widerspruch zur verbalen steht, in der Regel als die wahre, weil sie unwillkürlich geschieht. Sie ist verräterisch. So auch für Freud. Der Widerspruch zwischen dem, was in dem Kind vorgeht, und dem, was es sagt, äußert sich als semantische Inkongruenz zwischen außerverbaler und verbaler Mitteilung. Es kann sich durch Worte, aber nicht durch sein Gebaren verstellen.

Die gleiche Inkongruenz ist schon vorher, bei der Geburt der kleinen Schwester, zu beobachten. Verbal übernimmt Hans die Version der Eltern. Er hört die Mama im geschlossenen Nebenzimmer ,husten“ (stöhnen) und bemerkt: „Heut' kommt gewiss der Storch.“ Dieser Sprachregelung widerspricht aber, was er wahrnimmt, Arzt, Hebamme, Gefäße mit blutigem Wasser, und er macht zu allem „eine sehr mißtrauische, gespannte Miene“ (Freud 1969ff., VIII: 17).

„Die Inkongruenzen zwischen dem, was er sagt und dem, was er denkt“, die Freud (1969ff., VIII: 91) selbst für die Zeit der Phobie konstatiert, beginnen also schon am Ende der Vorgeschichte; sie sind ablesbar an der Inkongruenz zwischen den Worten und der Gebärdensprache und deuten auf eine psychische ,Spaltung“, wie Freud sie gleichzeitig in seiner Arbeit Über infantile Sexualtheorien (1908) beschrieben hat: „[...] die eine Meinung, mit der die Bravheit, aber auch die Sistierung des Nachdenkens verbunden ist, wird zur herrschenden bewußten; die andere, für die die Forscherarbeit unterdes neue Beweise erbracht hat, die nicht gelten sollen, zur unterdrückten ,unbewußten‘. Der Kernkomplex der Neurose findet sich auf diese Weise konstituiert“ (Freud 1969ff., V: 174f.; vgl. auch 163ff.). Lacan hat bekanntlich diese Spaltung des Subjekts, die „Entfremdung des Subjekts in seiner Rede“, für die ödipale Phase näher charakterisiert.

Für uns ist wichtig, dass Freud sie an der Sprache abliest und dass die Behinderung der begrifflichen Entwicklung des Kindes sich in einer Spaltung und Verzerrung seiner Sprache zu spiegeln scheint.

Wir kommen zum 2. Teil der Analyse, zum Prozess. Alfred Lorenzer übergeht seltsamerweise die sprachlichen Beobachtungen Freuds, obwohl er die Phobie des kleinen Hans, dieses „Abwehrgeschehen“, das die Angst vor dem Vater auf die vor den Pferden verlagert, überwiegend in linguistischen Kategorien, als „sprachliche Konfusion“ und als „semantische Verschiebung und Verfälschung der Namen“, beschreibt. Seine Interpretation:

Das Gesamtsymbol ,Vater` erfährt eine Bedeutungseinengung - bestimmte Anteile, so der umfallende Vater, der krawallmachende Vater, der fürchterlich starke Vater werden als bedrohlich ausgeklammert, desymbolisiert, aus dem Bewussten ausgeschlossen. In demselben Maße wird die Bedeutung ,Pferd“ ausgedehnt - verläuft die Symptombildung so, dass an Stelle der sprachlichen Objektrepräsentanzen des Vaterbildes das Symbol ,Pferd‘ einsteht. Der Konflikt ist damit vom Vater abgezogen, das Vaterbild als ganzes konfliktfrei geworden. 
Lorenzer betont nun, dass diese Bedeutungsverengung nicht an dem Wortgebrauch des kleinen Hans abgelesen werden könne: „Beide Bedeutungsänderungen entziehen sich jeder unmittelbaren Erfassung.“ „Im Begriff Pferd ist ein Bedeutungsanteil Vater enthalten und verborgen. Wenn wir sagen, dieser Bedeutungsanteil ist unbewußt, so heißt das auch, er ist aus der allgemeinen Kommunikation ausgeschlossen und entsprechend nicht in einem Sprachzeichen faßbar.“ Lorenzer bezeichnet die privaten Bedeutungen des Kindes als „nicht bewußt, also außerhalb der Sprache“, „nur indirekt aus den Verhaltensabweichungen als privatisiert“ ermittelbar, „vollkommen umgangssprachlich verschleiert“ (Lorenzer 1970: 97, 102). Wenn Lorenzers Voraussetzung einer semantischen Verschiebung und Verfälschung der Namen richtig ist, so ist die zweite Feststellung, sie sei ,nicht in einem Sprachzeichen fassbar‘, ziemlich unwahrscheinlich. Tatsächlich trifft sie nicht ganz zu. Nur muss man die Wörter nicht isoliert, sondern im Satzzusammenhang und im weiteren Kontext betrachten. Dem kleinen Hans ist der Bedeutungsanteil ,Vater' in seinem Begriff von ,Pferd“ nicht bewusst, und er wäre außerstande, diese Bedeutung zu artikulieren. Aber es gibt sprachliche und außersprachliche Indizien für seine privaten Bedeutungen. Das Unbewusste meldet sich an der sprachlichen Oberfläche als semantische Inkongruenz, die Rückschlüsse darauf zulässt, was das Kind ,eigentlich' meint.

Semantische Inkongruenz wird also zum diagnostischen Kriterium, zum Indiz, jetzt aber nicht die Inkongruenz zwischen Gebaren und Wort, sondern die semantische Unverträglichkeit innerhalb eines Satzes, einer Äußerung, die auffällige Verbindung von Sprachzeichen.

Ich nenne Beispiele solcher auffälligen Verknüpfungen, seien sie nun sachlich oder sprachlich ,unverträglich“:

\footnotetext{
Das Pferd wird ins Zimmer kommen. (Freud 1969ff., VIII: 27)

Da hab ich die Mammi ganz nackt im Hemde gesehen [...]. (Freud 1969ff., VIII: 33)

Der Wiwimacher wächst mit mir, wenn ich größer werde; er ist ja angewachsen. (Freud 1969ff., VIII: 35)

Er: „In der Nacht war eine große und eine zerwutzelte Giraffe im Zimmer, und die große hat geschrien, weil ich ihr die zerwutzelte weggenommen hab'. Dann hat sie aufgehört zu schreien, und dann hab' ich mich auf die zerwutzelte Giraffe draufgesetzt.“ Ich befremdet: „Was? Eine zerwutzelte Giraffe? Wie war das?“ Er: „Ja.“ (Holt schnell ein Papier, wutzelt es zusammen und sagt mir:) „So war sie zerwutzelt.“ (Freud 1969ff., VIII: 37)
}

Von den Verben gehen „Transfermerkmale“, Übertragungsmerkmale oder Projektionsmerkmale, auf das Subjekt oder Objekt über, mit denen sie verbunden sind: Großes, schreiendes X; kleines, zerwutzeltes, weggenommenes, besessenes X. Die Verben kreisen das Subjekt oder Objekt, das X ein, das hier zu substituieren wäre. - Freud (1969ff., VIII: 39) interpretiert den Text als eine Trutzphantasie des 
Kindes: „Schrei, soviel du willst, die Mammi [...] gehört mir“. Auffällig ist auch die folgende Stelle:

Hans: „Am meisten fürcht’ ich mich vor den Pferden, die am Munde so etwas haben.“ Ich: „Was meinst du? Das Eisen, das sie im Munde haben?“

Hans: „Nein, sie haben etwas Schwarzes am Munde (deckt seinen Mund mit der Hand zu).“

Ich: „Was, vielleicht einen Schnurrbart?“

Hans: „O nein!“ (Freud 1969ff., VIII: 46f.)

Freuds Kommentar:

Details, wie ich sie jetzt erfuhr, daß ihn besonders geniere, was die Pferde vor den Augen haben, und das Schwarze um deren Mund, ließen sich von dem aus, was wir wußten, gewiß nicht erklären. Aber als ich die beiden so vor mir sitzen sah und dabei die Schilderung seiner Angstpferde hörte, schoß mir ein weiteres Stück der Auflösung durch den Sinn, von dem ich verstand, daß es gerade dem Vater entgehen konnte. Ich fragte Hans scherzend, ob seine Pferde Augengläser tragen, was er verneinte, dann ob sein Vater Augengläser trage, was er gegen alle Evidenz wiederum verneinte, ob er mit dem Schwarzen um den ,Mund“ den Schnurrbart meinte, und eröffnete ihm dann, er fürchte sich vor dem Vater, eben weil er die Mutter so lieb habe. (Freud 1969ff., VIII: 41; ,Mund‘ an Stelle des kongruenten ,Maul‘ setzt Freud in Anführungszeichen)

Das erste Objekt der Furcht des kleinen Hans hat die Merkmale: groß, großer Wiwimacher, wird beißen, wird ins Zimmer kommen, hat etwas vor den Augen und etwas Schwarzes am,Mund“.

Ergänzt werden diese Merkmale durch eine parallele Phantasie, in der das große Objekt erfolglos schreit, weil der kleine Hans von dessen Partner Besitz ergreift.

Insgesamt ergeben diese Merkmale einen semantischen Umriss, in dem das Wort ,Vater` an Stelle der nicht kongruenten Wörter ,Pferd‘ bzw. ,Giraffe‘ als Lösung passt. Das Gefühl ihrer Richtigkeit teilt sich als plötzliches Kongruenzerlebnis mit. Das Lösungswort ,sitzt' und erhellt plötzlich das vorher nur dunkel Geahnte („,schoß mir ein weiteres Stück der Auflösung durch den Sinn“).

Das ist der Musterfall eines Rätsels. Volker Schupp (1972: 391ff.) hat in dem Nachwort zu seinem schönen Rätselbuch versucht, die Struktur des Rätsels linguistisch zu beschreiben. Der möglicherweise einfachste Typ ist das 33. Rätsel des Symphosios:

Ich habe rasende Zähne,

ich reiße Schafe,

suche blutige Beute und bluttriefende Nahrung.

Mit großem Grimm kann ich auch meine Stimme erheben.

Die Verben eröffnen in dem von ihnen bestimmten Satzplan Leerstellen und spezifizieren zugleich semantisch die von ihnen geforderten Ergänzungen. Eine 
Reihe von Prädikaten zeichnet in unserm Fall den semantischen Umriss des gesuchten Subjekts ,Ich‘. Es ist leicht zu erraten.

Ein zweiter Typ steht der Metapher nahe. Metaphern haben schon nach der Poetik des Aristoteles (Kap. 22) ein rätselhaftes und verräterisches Wesen: „Denn das ist das Wesen des Rätsels, wenn man Dinge sagt, die faktisch unmöglich miteinander zu verknüpfen sind. “Der Widerspruch gibt den Denkanstoß und führt im Durchschauen der Metapher, der Übertragung, zur Lösung. Auch dieser zweite Typ ist in der Sprache des kleinen Hans enthalten. Die ,schreiende Giraffe‘, die ,zerwutzelte Giraffe‘, der ,Mund des Pferdes‘ sind unbeabsichtigte Metaphern, ,Übertragungen', und der in ihnen steckende Widerspruch gibt den Hinweis auf das gesuchte Wort.

Die an dem Ersatzgegenstand beobachteten Merkmale kreisen das hinter ihm gesuchte Ding ein; der Symbolgedanke lässt, von diesen Merkmalen aus, nach analogen semantischen Merkmalen bei anderen Dingen suchen. Inkongruenzen im gegenwärtigen Kontext verweisen auf Kongruenzen, in denen der Sprachgebrauch berichtigt wäre. Alles zusammen liefert den semantischen Umriss, in den das Wort des Rätsels passt. Es leuchtet ein, wenn Freud sich immer wieder dieses Bildes bedient. So sehr szenisches Verstehen an der Analyse des kleinen Hans beteiligt sein mag, Freud bedient sich vielfach einer dem Puzzlespiel ähnelnden, an der sprachlichen Oberfläche orientierten linguistischen Deutetechnik. Er wertet semantische Inkongruenz als Indiz für Bedeutungsverschiebungen in der Sprache des Patienten und als Hinweis auf die Lösung des Rätsels, was „eigentlich“ gemeint sei.

\section{3}

Der Kleine Hans ist eine linguistische Kriminalgeschichte. Ist er auch eine Novelle (vgl. Timms 1983)? Die Antwort ist rasch zu geben. - In ungezählten Staatsexamina fragt der Kollege aus der Neueren Literaturgeschichte den armen Kandidaten: Ist Katz und Maus von Günter Grass noch eine Novelle? Was ist eine Novelle?

Zur Novelle gehört mit zuverlässiger Regelmäßigkeit die von Goethe sogenannte ,unerhörte Begebenheit' - meist ein tatsächliches Ereignis. Eine solche Begebenheit liegt in der Phobie des kleinen Hans vor.

Man betont die Nähe zur dramatischen Gattung. Wir haben sie ausführlich beschrieben. Nicht nur die eingelegten Dialogteile, die Protokolle, unterstützen den Eindruck des Dramatischen, sondern die Anlage des Ganzen. Sie ist nach Art eines Kriminalprozesses und nach dem Muster des sophokleischen Ödipus konzentriert auf einen einzigen Konflikt: Die Einleitung bietet dazu eine geraffte Exposition, der Hauptteil erfüllt mit seinen Wendungen der Angstphantasie, die 
als sprunghafter Befreiungsprozess erscheinen, die Forderungen einer konzentriert herausgearbeiteten Peripetie, und der Schluss lässt im Urteil den Konflikt abklingen, in der Art, dass er die Zukunft der Personen nur noch ahnungsvoll andeutet und nicht mehr gestaltet.

Die Kürze wird hervorgehoben, das begrenzte Personal der Novelle.

Der Verzicht auf das epische Ausmalen, auch auf das Ausmalen der Figuren.

Die straffe Architektur insgesamt wird betont, die strenge und gerade Linienführung auf einen Fluchtpunkt hin. Erzählen ist Auswahl. Hier ist das der Möglichkeit nach breite Erzählmaterial zwischen dem Rätsel ,Pferdephobie‘ und dem Wort des Rätsels ,Kleiner Ödipus' straff ausgespannt. Eine mehrfache Selektion hat stattgefunden. Der Vater, der als Schüler Freuds für Freud sein Kind beobachtet, und Freud, der aus dem vom Vater Eingereichten das für seine Deutung Wesentliche herauszieht, sichten das Material. Die Zeit tut es. Es ist deutlich eine Geschichte aus dem Wien vor 1914.

Gibt es in dieser Novelle den ,Falken', also das Leitmotiv oder Dingsymbol, das einheitsstiftend den Stoff organisiert? - Man könnte die Angstpferde nennen.

Die Novelle hat nicht selten einen Herausgeber und wird dadurch zur Rahmenerzählung - wie der Kleine Hans, in dem der kommentierende Herausgeber ein sehr starkes Gewicht hat.

Freud nennt den Kleinen Hans mit einem chemischen Begriff eine ,Analyse‘ Es ist die linguistische Kriminalnovelle eines Autors, der sich als Naturwissenschaftler versteht (vgl. den Aufsatz „Zur Terminologie der Psychoanalyse“ in diesem Band). Hans wird durch den biologischen Begriffsapparat erfasst, der in den Drei Abhandlungen zur Sexualtheorie ausgearbeitet ist, das Werk ist dadurch biologisch grundiert und erhebt einen naturwissenschaftlichen Wahrheitsanspruch. Sein Resultat ist für den Autor keine Überraschung, sondern zu erwarten, die Bestätigung eines Experiments.

Am Grunde komplexer psychischer Bildungen gebe es nur wenige Prozesse, heißt es am Schluss (Freud 1969ff., VIII: 122).

Der Gewinn, den Freud aus der naturwissenschaftlichen Sprache zog, lässt sich erkennen:

1. Er trat auf einem neuen, ungewissen, nicht ernst genommenen Gebiet in einen Sprachtyp ein, der das höchste Prestige genoss und mit dem die Autorität beweisbarer Wahrheit verknüpft war.

2. Die Umarbeitung des erotischen und sexuellen Bereichs in den biologischen Sprachtyp nahm diesen heraus aus den bisherigen Bewertungen, machte ihn zu einem neutralen, wissenschaftlichen Gegenstand, entzog ihn den Tabus. Die Rede von dem ,polygamen' und ,homosexuellen' kleinen Hans ist in dieser Hinsicht eine provozierende Umwertung. 
3. Die naturwissenschaftliche Sprache war, als Instrument, erprobt und hatte einen hohen sachaufschließenden Wert. Sie verpflichtete zu Genauigkeit und systematischer Beobachtung. Sie blieb aber, übrigens auch in den Augen Freuds, eine Bildersprache. Und sie muss es vermutlich aus grundsätzlichen Gründen bleiben. Das naturwissenschaftliche Selbstverständnis ist ein „Selbstmißverständnis“ (Habermas 1970: 262).

Mit der Aufgabe des naturwissenschaftlichen Paradigmas wird dann allerdings auch die ahistorische Konstanz des ödipalen Konflikts hinfällig. Warum dann nicht Orpheus? Paris? Warum nicht der ganze Wald der Mythologie? Die „Ableitung von nur wenigen Prozessen“ ist eine genuin naturwissenschaftliche Idee: sobald Freuds Lehre aus ihrer naturwissenschaftlichen Verpuppung befreit ist, ist diese Idee schon aufgegeben. Die Darstellung der Geschichte des kleinen Hans in den Bildern eines mit naturhafter Notwendigkeit verlaufenden Prozesses suggeriert fälschlich, der ,Prozess' der Analyse sei in der gleichen Weise, wie es die Naturvorgänge in den Augen der mechanistischen Naturwissenschaft vor 1900 waren, eine erweisbare Wahrheit. Die einzig wahre Sprache über die Angst des kleinen Hans wäre wohl doch nur die einer Novelle, die eingesteht, dass sie eine Novelle ist. In jedem Fall ist die naturwissenschaftliche Darbietungsform eine Metapher, Freud verlässt nirgends die geisteswissenschaftliche Disziplin - was er darstellt, ist, so oder so, ein Grenzfall der Literatur. 